\title{
Achieving boosted expansion of transplantable hematopoietic stem cells by co-culture with autologous endothelial cells
}

\author{
Ayodele O Ilesanmi* \\ Kwara State University, Malete, Kwara State, Nigeria
}

\begin{abstract}
Currently, hematopoietic stem cells derived from the bone marrow or from cord blood are used to treat patients who require hematopoietic stem cell treatment. In spite of the varied sources from which hematopoietic stem cells may be obtained, there is still shortage of genetically matched hematopoietic stem cell grafts. As a result, many patients requiring transplantation cannot benefit from the procedure. Efforts have been made in time past to expand this unique type of adult stem cells in culture, with little success. These stem cells, even in the presence of several exogenous cytokines, serum factors and support from (mesenchymal) cells do not propagate the stem cells beyond a few days. However, reports from recent studies indicate that long-term propagation and large-scale expansion of hematopoietic stem cells is now possible with the use of endothelial cells in co-culture with hematopoietic stem cells. These studies have demonstrated that the presence of endothelial cells is necessary for promoting self-renewal of Hematopoietic Stem Cells (HSC), for long-term survival of repopulating HSCs and for increasing the number of these cells in culture. Endothelial cells from blood vessels constitute the basic building blocks of the human vasculature, being a key constituent of the bone marrow and vascular niche that support hematopoietic mobilization, maintenance and regeneration. Researchers have not only found that endothelial cells could greatly expand HSCs but the proliferation induced by endothelial cells carries no risk of tumor formation. There is a dire need to translate this revolutionary finding from the laboratory into routine clinical use. The purpose of this review therefore, is to glean the several studies related to the subject into a practical protocol scalable for clinical application.
\end{abstract}

\section{Introduction}

Hematopoietic stem cells are very unique in their growth requirements, demanding an adequate microenvironment similar to that in the bone marrow to maintain their stemness. While in vivo bone marrow niche conditions have made transplantation possible, enabling survival and repopulation of HSCs, the same cannot be said of in vitro attempts to expand these unique cells to obtain large numbers ex vivo for transplantation. Most attempts at expanding hematopoietic stem and progenitor cells (HSPC) ex vivo, for enhanced in vivo engraftment in patients have been clinically unsuccessful because of poor yields with regard to cell numbers, or inappropriate early differentiation of the HSPC starting cell population. Recent attempts at achieving HSC growth and sustenance have even got to the extent of creating synthetic 3 -dimensional matrixes in order to mimic the bone marrow niche.

The ultimate goal of cord blood and bone marrow HSC expansion is to generate clinically safe, transplantable HSCs, which can rescue the recipient's blood system. To be efficacious, the graft should contain sufficient numbers of both the Long-Term Reconstituting Hematopoietic Stem Cells (LT-HSCs) and progenitor cells. Generally, ex vivo expansion of HSCs has been advocated in order to increase graft number. Several investigators have premised HSC expansion largely on the use of exogenous growth factors, although this approach has, to date, not given satisfactory results. For instance, Yao et al. [1] proposed the use of IMDM, cytokine cocktails and serum substitutes without the use of stromal cells. Expansion of HSCs with this combination only achieved limited success. The substitution of natural serum has eliminated a rich source of vital ingredients for HSC culture. Bovine sera contain basic nutrients and proteins like albumin which is capable of transporting other vital molecules into the cell
$[2,3]$. Human serum or plasma, for instance, has been known to be a good preservative for HSPCs. So also, is albumin that is contained in the serum [4]. Serum albumin binds to several small molecules and acts as an antioxidant in most cases [5], resulting in the regulation of a range of processes, such as apoptosis (negative regulation), distribution of cellular components, cellular response to starvation as well as chaperone binding [2]. However, the use of animal serum in growing cells meant for transplantation to humans has been a subject of serious controversy for a long time because of the associated risks. Serum-free media obviates the risk of infection from viruses, variation between individual batches and regulatory restrictions. In addition, serum may contain inhibitors of cell proliferation. Moreover, the use of a serumfree media in cell expansion also makes the medium standardized since it is then chemically defined. Other supplemental growth factors/ cytokines are usually obtained from exogenous sources and being synthetic substances, do not adequately represent the HSC niche. Culture conditions with stroma cell constituents may have the required appropriate amounts of these cytokines released into cell culture endogenously, an advantage that stroma-containing cultures have over stroma-free cultures. The crosstalk between the cultured cell and the conditioning cell is also of tremendous advantage to propagation. In

${ }^{\star}$ Correspondence to: Ayodele O Ilesanmi, Kwara State University, Malete, Kwara State, Nigeria, E-mail: ayodeleilesanmi2002@yahoo.com

Key words: haematopoietic stem cells, expansion, endothelial cells, culture media, stromal vascular fraction, co-culture

Received: October 19, 2018; Accepted: October 30, 2018; Published: November 02,2018 
stroma-free culture systems, SCF and Flt3-ligand maintain HSCs while TPO supports the expansion of both short- and long-term repopulating HSCs [6]. It may be possible for these cells to be significantly expanded using this formulation, although the maintenance of the cells in that state is much more difficult with only these cytokines. As a result, normal HSCs cannot be cultured for more than a few days without losing their stem cell properties. The cells thus differentiate and are no longer able to proliferate. Recent efforts have therefore focused on the use of cells that are able to generate these growth factors endogenously while in co- culture with HSCs and which sustain in vitro growth, expansion and engraftment in patients for longer periods. Several publications have documented successful amplification of Hematopoietic Stem/ Progenitor Cells (HSPCs) on endothelial or mesenchymal cells [7-11]. This concept is based on the premise that these two types of cells are part of the population of cells making up the hematopoietic stem cell niche in the bone marrow and which provide the cues needed by the HSCs for growth and proliferation.

Bone marrow Mesenchymal Stem Cells (MSC) participate in the bone marrow microenvironment by providing growth factors and matrix proteins, which all act to regulate blood production, through cell-to-cell interactions. MSCs have been demonstrated to improve expansion of cord blood Hematopoietic Stem Cells (HSC) in several studies $[7,9,12]$. However, while expansion has been possible with MSCs, the long-term propagation as well as the engraftment of HSCs grown in co-culture with MSCs after transplantation, has been hampered [12]. Raynaud et al. [13] investigated the ability of different feeder layers including mesenchymal progenitors and endothelial cells to expand hematopoietic stem and progenitor cells using a competitive assay. They found that HSPCs had preference for endothelial cells in vitro. It has also been suggested that plastic-adherent bone marrow stromal cells may be inadequate to preserve primitive HSCs [11].

In addition to other preliminary findings, studies by Butler et al. [14] have shown the potent capacity of endothelial cells to greatly boost hematopoietic stem cell expansion on a large scale, without the addition of exogenous growth factors and serum [10,14]. In these studies, they found that Endothelial Cells (ECs) were necessary for the long-term self- renewal of long-term repopulating HSCs. Noting that the maintenance of HSCs in steady-state condition is physiologically different from a situation in which the bone marrow is reconstituted after myeloablation; they inferred that the latter condition is similar to growing and expanding HSCs in vitro. The studies affirmed their postulation that the vascular niche of the bone marrow microenvironment is responsible for balancing the expansion and differentiation of HSCs through the expression of angiocrine factors that prevent the exhaustion of the repopulating long- term HSC pool. It is evident that these angiocrine factors from the vascular niche are secreted by no other cells than the endothelial cells (Figure 1).

\section{Endothelial cells as a key factor in long-term expansion and engraftment of HSCs}

Hematopoiesis is regulated majorly by the vascular niche in which endothelial cells reside. Some of the functions of the vascular niche include hematopoietic differentiation, mobilization and maintenance of the HSC in or outside the bone marrow. In addition, endothelial cells play an active part in hematopoietic regeneration $[6,15]$. To prove that endothelial cells are the key to long-term renewal of hematopoietic stem cells and large-scale expansion of HSCs, Butler et al. [14] carried out a study using an animal model for an in vivo transplantation of HSCs grown in co-culture with ECs that had earlier been transfected

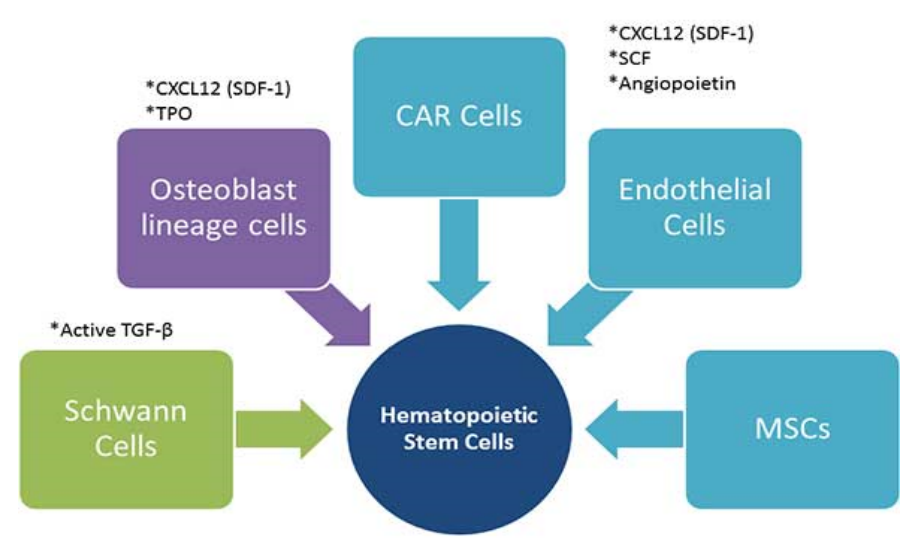

Figure 1. Bone marrow stromal cells and some of the cytokines they secrete for HSC proliferation

with an adenovirus. The ECs used were isolated from the umbilical vein obtained from a human source before being transfected with a lentivirus; and the CD34+ cells used for co-culture were also from human cord blood, possibly of autologous source. The ECs were first depleted by positive selection with anti-CD45 antibody bound to microbeads. Apart from using the monoclonal anti-CD45 antibody and other cell markers, cells were also analyzed by flow cytometry. The resulting ECs (E4ORF1+) were such that could make possible the avoidance of supplementation with cytokines or serum while expanding HSCs on EC co-culture, without compromising their proliferative and angiogenic properties. They initially interrogated the transfected ECs against KLS 1000 (HSC) cells with supplementation of only sKitL, comparing the expansion obtained with that obtained when ordinary ECs from Human Umbilical Vein Endothelial Cells (HUVEC) were used in co-culture without supplementation. The idea was to establish that the transfected ECs (E4ORF1+ ECs) provide a unique vascular platform that not only maintains but also stimulates longterm expansion of true repopulating HSCs in the absence of serum and cytokine supplementation, thus simulating the bone marrow vascular niche that was required for the long-term in vivo expansion of HSCs.

Some of the relevant take-away from this study include the following: HSCs cultured with exogenous cytokines alone do not support long-term engraftment of HSCs since they may contain fewer number of long-term repopulating HSCs; ECs activate Notch signaling on co-cultured HSCs which then promote self-renewal of HSCs by reducing exhaustive differentiation into progenitors that are lineagecommitted and the degree of Notch signaling on the HSCs increases over time when in co-culture with ECs; Lack of direct cellular contact with ECs resulted in poor survival and inefficient expansion of longterm HSCs. In addition to all these, it was also found that blocking the angiogenic signaling (by inhibiting the activities of VE-cadherin and VEGFR2) resulted in decreased number of long-term HSCs in co-culture with ECs. Taken together, it is evident that endothelial cells have a central role to play in the maintenance and long-term expansion of not just the hematopoietic progenitor cells, but particularly of longterm repopulating HSCs. To further buttress the latter notion, an in vivo transplantation assay of the HSCs was carried out on an animal model in the said study. The in vivo transplantation assay monitored with Green Fluorescent Protein (GFP) was meant to observe the expansion of only long-term HSCs available in the expanded population of cells and not the ECs. It was discovered that one out of four expanded cells were long-term repopulating cells from the 21-day EC-supported culture, four months post-transplantation, confirming 
that ECs and not other factors were responsible for the large-scale expansion of long-term HSCs. Successful serial transplantation assay of the expanded HSCs in the animal model also suggests that ECs are essential for the long-term engraftment of transplanted HSCs. The study therefore showed the superior ability of ECs to boost the expansion of long-term, engrafting HSCs along with the progenitor cells. Mizer et al. [16] reported that direct injection of endothelial cells into animal subjects resulted in stimulating hematopoietic activity, in addition to expanding the number of functional HSCs ex vivo in other instances. Human endothelial cells are therefore an excellent platform for the ex vivo expansion of HSCs as well as for supporting in vivo treatment of hematological disorders.

\section{Summarized procedure for obtaining autologous ECs for co-culture}

Endogenously-derived endothelial cells may be derived in large numbers by the protocol described earlier in a previous publication [17]. The EC cells are obtained from the same individual that is to be transplanted with the HSCs. Vascular tissue endothelial cells may be relatively easily obtained by just processing fatty tissue in the laboratory. Although endothelial cells may also be harvested from the peripheral circulation, the number that can be obtained is incomparable. Moreover, circulating endothelial cells are products of injurious processes in the vascular endothelium and may be limited in their potentials for enhancing cell proliferation. Going by the procedure suggested; fatty tissue from the patient is collected by liposuctioning and processed to obtain either Stromal Vascular Fraction (SVF) or Vascular Fraction Cells (VFC) by a technology known as ultrasonic cavitation. For the purpose of HSC co- culture, the latter product is to be preferred. The resultant Vascular Stromal Cells (VSCs) may then be processed further in the laboratory to selectively obtain large numbers of endothelial cells; possibly with minor contamination from other stromal cells which themselves are useful in the intended culture. By so doing, the use of autologous ECs will achieve two purposes: one, the final HSC product is prevented from cross- contamination from allogeneic cells and again, expansion of the patient's HSCs is greatly boosted in culture by the presence of the autologous ECs.

In order to use the ECs for co-culture with HSCs, the addition of soluble kit ligand to the culture medium is recommended. This supplement is capable of increasing the expansion yield of HSCs in coculture with ECs. Soluble Kit Ligand (sKitL) is added at a concentration of $10 \mathrm{ng} / \mathrm{ml}$ to the culture medium intended for HSC co-culture. This supplement synergizes with ECs to expand HSCs by two mechanisms: by increasing the survival of HSCs and also by inducing the release of VEGF-A, which subsequently induces surface expression of Jagged-2 on the ECs $[10,18]$. In other words, ECs that sustain the HSCs are themselves maintained because sKitL activates the ECs to release a growth factor vital to expansion and in addition promotes survival.

\section{Proposed protocol for HSC co-culture with endothelial cells}

Expansion of cord blood CD34+ cells with SCF, Flt3-ligand, IL6, VEGF and IL-1, in experiments with or without autologous coculture system using Umbilical Vein Endothelial Cells (HUVEC) has demonstrated that co-culture system was superior to liquid culture $[10,19]$. Therefore, the use of co-culture is to be preferred over that of conditioning medium. For the co-culture process, $4 \times 105$ endothelial cells/well is seeded in 6-well microplates. In the Butler et al. study, the initial EC population in culture before the introduction of hematopoietic stem/ progenitor cells was $2 \times 10^{6}$, while the number of HSPCs seeded into medium as co-culture was $5 \times 10^{4}$ cells, a ratio of about 40 ECs to one HSPC [10]. Endothelial cells along with trace amounts of other stromal cells constitute the feeder layer in this case. The culture medium should consist of X-vivo serum-free media (or any other appropriate HSC expansion medium, as there are now many of them available in the market), supplemented with $10 \mathrm{ng} / \mathrm{ml}$ soluble Kit Ligand (sKitL). Most HSC expansion media available in the market contain optimal amounts of key soluble growth factors necessary for the basic cultivation of hematopoietic stem and progenitor cells. These basic growth factors include: SCF, TPO, Flt-3 ligand, IL-6 and c-kit $[6,20]$. sKitL is however required to prevent the apoptosis of primary ECs which occur in the absence of serum and cytokines [14]. Although several other exogenous cytokines have been identified as being capable of enhancing HSC expansion, it is obvious that not all growth factors found experimentally suitable can be incorporated into a commercial expansion medium. To do so would make the cost of expansion media out of reach. Moreover, the presence of endothelial cells in co-culture obviates the need to use a large array of cytokines.

Exactly $1.5 \mathrm{ml}$ of the culture medium is layered on the endothelial cells in the well. Using the ratio of ECs to HSPC as in Butler et al. [14] study, $1 \times 10^{4}$ immunomagnetic-sorted, normal peripheral blood CD34+ hematopoietic cells from G-CSF mobilized donors or from cord blood -are then plated in direct contact with the ECs and stromal layers. However, since up to $50 \%$ of SVF is constituted by CD34+ cells (exact percentage could be up to 63\%) [21], the SVF itself may be selected directly with immunomagnetic beads to extract the CD34+ cells required for seeding culture, making patient mobilization with G-CSF quite unnecessary; that is in a situation where the ECs are harvested from SVF processing. Harvested CD34+ cells so obtained may then be counted by flow cytometer. Co-cultures in which HSCs are in direct contact with ECs should produce a bumper yield, as was found by Butler et al. [10]. The cultures are maintained initially for 7 days at $37^{\circ} \mathrm{C}$ in a humidified atmosphere at $5 \% \mathrm{CO}_{2}$ and $5 \% \mathrm{O}_{2}$ tension with twice weekly media changes [22]. Early harvesting of cells at 7 days is to ensure that cells do not begin to differentiate before the next passage. The $5 \%$ oxygen tension requirement is to make room for cytokine secretion by ECs since growth factor secretion is increased under hypoxic conditions and for the hypoxic requirement of HSCs as is found in their bone marrow quiescent state [23]. According to Zhang and Sadek, in vivo studies have confirmed that most quiescent and primitive HSCs reside in hypoxic bone marrow regions with diminished blood perfusion [24]. Media change is constituted by replacement with fresh cultivation medium in the ratio 1:2 of stale medium (conditioning medium) to fresh expansion medium. This will ensure that inhibitory waste products from culture are diluted out; while the concentration of depleted growth factors is restored. Xie and Zhang, suggested that decreasing the concentration of accumulating negative factors and increasing culture volume to maintain a lower cell density, favor HSC expansion [25]. The process involves aspirating conditioning media from the wells without affecting the layer of endothelial cells and adding two volumes of fresh expansion medium to one volume of stale medium. The aspirated media should contain a number of expanded HSCs. The initial plated well is intended for sub-culturing (or replating) after the second media change. It is only after replating that cells are allowed to grow to confluence and complete its growth cycle of three weeks. After seven days of culture, just before the second media change is due, the cells are harvested from both the supernatant and the stroma of the culture, while a sample from it is counted again by flow cytometer. The cells from each well are centrifuged at $400 \mathrm{~g}$ for 
5 minutes at $4^{\circ} \mathrm{C}$ in a conical tube in a sterile environment. By this time, at least a ten-fold increase would have occurred and the potential for marked differentiation is greatly diminished. The pellet of cells are recovered and transferred into a T25 flask containing expansion medium with the autologous endothelial cells again serving as feeder layer, on which the HSCs are overlaid. Considering the volume of the flask, the expansion envisaged now is ten times that which could have been obtained in micro-plate wells of $1.5 \mathrm{ml}$ capacity, although the number obtained could even be more. The volume of expansion media, the numbers of both ECs and the HSCs to be seeded into culture are also appropriately increased ten-fold. Thus approximately $4 \times 10^{6} \mathrm{ECs}$ are seeded into the T25 flask to which $1 \times 10^{5}$ HSCs harvested from the primary culture are layered upon, within the T25 flask. While the T25 flask has a capacity of $25 \mathrm{cc}$, a maximum of $13.5 \mathrm{ml}$ of expansion media is added to allow carbon dioxide penetration into the culture flask. What this means is that from the six microplate wells, one can expand the harvested HSCs further in six 25-T flasks or even T75 flasks, bearing in mind the number ratio used initially for cultivation. The growth conditions are then maintained as previously described. After growing to confluence, cells are harvested by centrifugation while adhering to protocols previously outlined. The expanded cells are then pooled and washed in several changes of medium and kept in a sterile atmosphere for clinical use. A sample of the cells is taken for cell count and viability, while a portion of the cells is replated in methylcellulose in order to determine the number of Colony-Forming Cells (CFC). The most critical aspect of this procedure is that aseptic precautions must be maintained throughout every step of the process.

\section{Counting the numbers}

By the time confluence is reached, each flask would have the cells expanded by at least one hundred and fifty-fold. Thus, each T25 flask should now contain $150 \times 10^{5}$ HSCs. In six (T25 flask) places, a figure of $900 \times 10^{5}$ is generated. If T75 flasks are used, the figure is multiplied by three. To generate this number of HSCs, we would need six times of $\left(4 \times 10^{5}\right)$ ECs for the microplate wells plus six times of $\left(4 \times 10^{6}\right)$ ECs for the T25 flasks, amounting to $2.4 \times 10^{6}$ plus $2.4 \times 10^{7}$ (or a total $2.64 \times$ $10^{7}$ ) ECs. It is possible to harvest about a billion SVF cells from $100 \mathrm{cc}$ of fat tissue. A minimum $7 \%$ of the SVF being ECs would yield $7 \times 10^{7}(7 \%$ of one billion) endothelial cells - about three times of what is required. Since there are more ECs generated than would be used, more culture flasks could be enlisted. For instance, the T25 flasks content may be further expanded in T75 flasks, and three times the cell yield in T25 flasks would be obtained.

This protocol should generate at least $1 \times 10^{8}$ cells with only one passage and this should be sufficient to enable engraftment in the patient. Additionally, several millions of HSC primary cells may still be available from processing the SVF that could supplement the number obtained from culture. More importantly, a good fraction of this population of cells would be long-term repopulating HSCs, capable of enabling multilineage engraftment. If more HSCs are desired, the harvested ECs have to be increased proportionally; and this begins with the volume of fat tissue harvested for processing. Fortunately, as much as 350cc of fat may be harvested by lipoaspiration. Ideally, bone marrow and peripheral blood mobilized stem cell grafts should contain approximately $10^{8} \mathrm{CD} 34+$ cells, while cord blood graft may contain as few as $5 \times 10^{6} \mathrm{CD} 34+$ cells. Although the number appropriate for SVF grafts has not been determined, since bone marrow, peripheral blood and SVF are adult tissues, the outcome using the same number of CD34+ cells in graft should be similar.
One challenge to be faced in the expansion of HSCs is that of being able to characterize the product obtained from co-culture. Although most stem cell researchers agree that HSCs express CD34+, CD90+ and CD133+ antigens on their surfaces, it has been noted that cultured HSCs have a tendency for exhibiting altered surface phenotypes [26]. To date, no known reliable biomarker exists that can be quickly used for assessing the long-term activity of both the HSCs expanded ex vivo and those obtained directly from the bone marrow or peripheral blood without manipulation. The only known reliable method for assessing HSCs for long-term activity remains the time-consuming serial transplantation assay in animal models. Fares et al [27] recently came up with a biomarker for characterizing HSCs obtained from cord blood. Ironically, this marker, also known as Endothelial Protein C Receptor (EPCR) was initially found to characterize HSCs in murine samples but has now been found to be of utility in identifying longterm activity in human cord blood samples. It is hoped that its ability to assess HSCs in humans will extend to adult bone marrow and peripheral blood samples.

In order to achieve the goal of attaining sufficient transplantable cells that could yield clinically satisfactory engraftment, the expansion process must in addition to maintaining appropriate culture conditions, be mindful of the number factor and the ratios, from the beginning to the end.

\section{Conclusion}

This review is important because there is as yet no easy solution to expanding hematopoietic stem cells ex vivo although the demand for HSC transplantation that is safe and efficacious is increasing by the day. Researchers who have hitherto focused on the use of mesenchymal stem cells and cytokines to expand HSCs now have a better, highyielding alternative feeder cells to work with by employing recently promoted technologies to extract endothelial cells that can be used in co-culture, to expand HSCs into large numbers.

This review advances the field by proposing the use of a readily available and abundant source of endothelial cells in body tissues for growing and expanding HSCs. The use of autologous endothelial cells that could be obtained with relative ease, to boost the expansion of HSCs is not only feasible but ECs can be made available in sufficient numbers, for long-term sustainable HSC co-culture as to generate enough HSCs that include a relatively large proportion of long-term, reconstituting HSCs and which number is adequate for effective transplantation.

\section{References}

1. Yao CL, Hsu SC, Hwang SM, Lee WC, Chiou TJ (2013) A stromal-free, serum-free system to expand ex vivo hematopoietic stem cells from mobilized peripheral blood of patients with hematologic malignancies and healthy donors. Cytotherapy 15: 1126-

2. 135. [Crossref]

3. Tekkatte C, Gunasingh GP, Cherian KM, Sankaranarayanan K (2011) "Humanized" stem cell culture techniques: the animal serum controversy. Stem Cells Int 2011 504723. [Crossref]

4. Arora M (2013) Cell Culture media: A review. Mater Methods 3: 175.

5. Koestenbauer S, Zisch A, Dohr G, Zech NH (2009) Protocols for hematopoietic stem cell expansion from umbilical cord blood. Cell Transplant 18: 1059-1068. [Crossref]

6. Taverna M, Marie AL, Mira JP, Guidet B (2013) Specific antioxidant properties of human serum albumin. Ann Intensive Care 3. [Crossref]

7. Shatirishvilli G, Mardaleishvili K, Loladze G (2014) Cord Blood Hematopoietic Stem Cell Expansion: Preclinical Studies and Clinical Trials. CellR4 2: e1209.

8. Zhang Y, Chai C, Jiang XS, Teoh SH, Leong KW (2006) Co-culture of umbilical cord blood CD34+ cells with human mesenchymal stem cells. Tissue Eng 12: 2161- 2170. [Crossref] 
9. Li N, Eljaafari A, Bensoussan D, Wang Y, Latger-Cannard V, et al. (2006) Human umbilical vein endothelial cells increase ex vivo expansion of human CD34(+) PBPC through IL-6 secretion. Cytotherapy 8: 335-342. [Crossref]

10. Li N, Feugier P, Serrurrier B, Latger-Cannard V, Lesesve JF, et al. (2007) Human mesenchymal stem cells improve ex vivo expansion of adult human CD34+ peripheral blood progenitor cells and decrease their allostimulatory capacity. Exp Hematol 35: 507-515. [Crossref]

11. Butler JM, Gars EJ, James DJ, Nolan DJ, Scandura JM, et al. (2012) Development of a vascular niche platform for expansion of repopulating human cord blood stem and progenitor cells. Blood 120: 1344-1347. [Crossref]

12. Isern J, Marti'n-Antonio B, Ghazanfari R, Marti'n AM, Lo'pez JA, et al. (2013) self- renewing human bone marrow mesenspheres promote hematopoietic stem cell expansion. Cell Reports 3: 1714-1724. [Crossref]

13. de Lima M, McNiece I, Robinson SN, Munsell M, Eapen M, et al. (2012) Cord-blood engraftment with ex vivo mesenchymal-cell coculture. $N$ Engl J Med 367: 2305-2315. [Crossref]

14. Raynaud CM, Butler JM, Halabi NM, Ahmad FS, Ahmed B, et al. (2013) Endothelia cells provide a niche for placental hematopoietic stem/progenitor cell expansion through broad transcriptomic modification. Stem Cell Res 11: 1074-1090. [Crossref]

15. Butler JM, Nolan DJ, Vertes EL, Varnum-Finney B, Kobayashi H, et al. (2010) Endothelial Cells Are Essential for the Self-Renewal and Repopulation of NotchDependent Hematopoietic Stem Cells. Cell Stem Cell 6: 251-264. [Crossref]

16. He N, Zhang L, Cui J, Li Z (2014) Bone marrow vascular niche: home for hematopoietic stem cells. Bone Marrow Res 2014: 128436. [Crossref]

17. Mizer JC, Ichim TE, Alexandrescu DT, Dasanu CA, Ramos F, et al. (2012) Exogenous endothelial cells as accelerators of hematopoietic reconstitution. $J$ Transl Med 10: 231. [Crossref]
18. Ilesanmi AO (2018) A novel way of harvesting a large population of endothelial cells for clinical and experimental use. Biomed Res Rev 2: 120

19. Heissig B, Hattori K, Dias S, Friedrich M, Ferris B, et al. (2002) Recruitment of stem and progenitor cells from the bone marrow niche requires MMP-9 mediated release of kit-ligand. Cell 109: 625-637. [Crossref]

20. Yildrim S, Boehmler AM, Kanz L, Mohle R (2005) Expansion of cord blood CD34+ hematopoietic progenitor cells in co-culture with autologous umbilical vein endothelia cells (HUVEC) is superior to cytokine-supplemented liquid culture. Bone Marrow Transplant 36: 71-79. [Crossref]

21. Andrade-Zaldivar, H, Santos L, Rodriguez AL (2008) Expansion of human hematopoietic stem cells for transplantation: trends and perspectives. Cytotechnology 56: 151-160. [Crossref]

22. Bersenerv A (2013) Breaking down fat: composition of stromal vascular fraction Stem cell assays.

23. Glettig DL, Kaplan DL (2013) Extending Human Hematopoietic Stem Cell Survival In Vitro with Adipocytes. Biores Open Access 2: 179-185. [Crossref]

24. Oberbauer E, Steffenhagen C, Wurzer C, Gabriel C, Redl H, et al. (2015) Enzymatic and non-enzymatic isolation systems for adipose tissue-derived cells: current state of the art. Cell Regen 4: 7. [Crossref]

25. Zhang CC, Sadek HA (2014) Hypoxia and metabolic properties of hematopoietic stem cells. Antioxid Redox Signal 20: 1891-1901. [Crossref]

26. Xie J, Zhang C (2015) Ex vivo expansion of hematopoietic stem cells. Sci China Life Sci 58: 839-853. [Crossref]

27. Notta F, Doulatov S, Laurenti E, Poeppl A, Jurisica I, Dick JE (2011) Isolation of single human hematopoietic stem cells capable of long- term multilineage engraftment. Science 333(6039): 218-221.

28. Fares I, Chagraoui J, Lehnertz B et al. (2017) EPCR expression marks UM171expanded CD34 cord blood stem cells. Blood 129(25): 3344- 335.

Copyright: (C2018 Ilesanmi AO. This is an open-access article distributed under the terms of the Creative Commons Attribution License, which permits unrestricted use, distribution, and reproduction in any medium, provided the original author and source are credited. 